\title{
Karga Arama Algoritması İçin Parametre Analizleri
}

\author{
Emine BAŞ ${ }^{1 *}$ \\ ${ }^{1 *}$ Selçuk Üniversitesi, Kulu Meslek Yüksekokulu, Bilgisayar Teknolojileri Bölümü, Konya, Türkiye, (ORCID: 0000-0003-4322-6010), emineozcan@selcuk.edu.tr
}

(International Conference on Design, Research and Development (RDCONF) 2021 - 15-18 December 2021)

(DOI: $10.31590 /$ josat.1039646)

ATIF/REFERENCE: Baş, E., (2021). Karga Arama Algoritması için Parametre Analizleri. Avrupa Bilim ve Teknoloji Dergisi, (32), 878-882.

Öz

Bu makale, kargaların akıllı davranışına dayanan, Karga Arama Algoritması (KAA) adlı yeni bir metasezgisel algoritmayı tanıtmaktadır. KAA, kargaların fazla yiyeceklerini saklanma yerlerinde sakladığı ve yiyecek gerektiğinde geri aldığı bu fikirden yola çıkarak çalışan popülasyona dayalı bir tekniktir. KAA metodu üzerinde fl sabit parametresi lokal ve gobal arama yeteneği arasında önemli farklılıklar yaratmaktadır. Bu çalışmada beş farklı fl değeri belirlenmiş ve KAA ' nın performansı üzerindeki etkisi araştııılmıştır. KAA ile on farklı son yıllarda geliştirilmiş CEC-C06-2019 seri fonksiyonları çözülmüştür. KAA ile çeşitli sonuçlar elde edilmiştir (ortalama, standart sapma, en iyi ve en kötü). KAA ile elde edilen sonuçlar birbirleri ile ve çeşitli sezgisel algoritmaların sonuçları ile karşılaştırılmıştır. Test sonuçları, KAA kullanımının diğer algoritmalara kıyasla umut verici sonuçlar bulmasına yol açabileceğini ortaya koymaktadır.

Anahtar Kelimeler: Karga Arama Algoritmas1, Sürekli optimizasyon, CEC-C06-2019.

\section{Parameter Analysis for Crow Search Algorithm}

\begin{abstract}
This paper introduces a new metaheuristic algorithm named Crow Search Algorithm (CSA) based on the intelligent behavior of crows. CSA is a population-based technique that works from this idea that crows store their excess food in their hiding places and retrieve it when needed. On the CSA method, the constant parameter fl creates significant differences between local and global search capabilities. In this study, five different fl values were determined and the effect of CSA on performance was investigated. CEC-C06-2019 serial functions developed in ten different recent years have been solved with CSA. Various results were obtained with CSA (mean, standard deviation, best and worst). The results obtained by CSA were compared with each other and with the results of various heuristic algorithms. The test results reveal that the use of CSA can lead to promising results compared to other algorithms.
\end{abstract}

Keywords: Crow Search Algorithm, Continuous optimization, CEC-C06-2019.

\footnotetext{
* Sorumlu Yazar: emineozcan@selcuk.edu.tr
} 


\section{Giriş}

Metasezgisel algoritmalar, son derece doğrusal olmayan ve çok modlu olan çoğu gerçek dünya optimizasyon problemini çözmek için umut verici bir performans göstermektedir. Tüm meta-sezgisel algoritmalar, belirli bir rastgeleleştirme ve yerel arama dengesi kullanır. $\mathrm{Bu}$ algoritmalar zor optimizasyon problemleri için iyi çözümler bulabilir, ancak optimal çözümlere ulaşılabileceğinin garantisi yoktur. Bu algoritmaların çoğu zaman işe yaraması umulmaktadır, ancak her zaman bunu garantilemez. Metasezgisel algoritmalar küresel optimizasyon için uygun olabilir. Tüm modern doğadan ilham alan yöntemlere metasezgisel şekklinde isim verilmektedir (Yang, 2011).

Mevcut eğilim, zor problemlerin üstesinden gelmek için doğadan ilham alan metasezgisel algoritmaları kullanmaktır ve metasezgisellerin şaşırtıcı bir şekilde çok verimli olduğu gösterilmiştir. Bu nedenle, metasezgisel literatür son yirmi yılda muazzam bir şekilde genişlemiştir. İyi bilinen metasezgisel algoritmalardan bazıları şunlardır: doğal seleksiyona dayalı genetik algoritma (GA), kuş sürüsünün sosyal davranışına ve balık sürüsüne dayalı parçacık sürü optimizasyonu (PSO), müzik doğaçlama sürecine dayalı armoni araması (HS), bazı guguk kuşu türlerinin kuluçka parazitliğine dayalı guguk kuşu arama algoritması, mikro yarasaların ekolokasyon davranışına dayalı yarasa algoritması (BA), hayvan arama davranışına dayalı grup arama optimize edicisi (GSO), tropiklerin yanıp sönen 1 ş1k modellerine dayalı ateş böceği algoritması (FA) vb. Bu makalede tanitılan metasezgisel yöntem optimizasyon problemlerini çözerken umut verici sonuçlar elde edebileceğimiz kullanıcı dostu (basit konsept ve kolay uygulama) bir metasezgisel tekniktir.

Kargalar, şu anda dünyanın en zeki hayvanları arasında sayılan, yaygın olarak dağılmış bir kuş cinsidir. Bir grup olarak, kargalar dikkat çekici zeka örnekleri gösterirler ve zeka testlerinde genellikle çok yüksek puanlar alırlar. Yüzleri ezberleyebilir, araçları kullanabilir, karmaşık yollarla iletişim kurabilir ve mevsimler boyunca yiyecekleri saklayabilir ve alabilirler (Askarzadeh, 2016).

Bir karga sürüsü, optimizasyon süreciyle pek çok benzerliği olan bir davranış sergilerler. Bu davranışa göre kargalar fazla yiyeceklerini ortamın belirli konumlarında (saklanma yerlerinde) saklarlar ve depolanan yiyecekleri gerektiğinde geri bulurlar. Kargalar daha iyi besin kaynakları elde etmek için birbirlerini takip ettikleri için açgözlü kuşlardır. Bir karganın gizlediği besin kaynağını bulmak kolay bir iş değildir, çünkü bir karga onu takip eden başka bir kargayı görürse, karga ortamın başka bir pozisyonuna giderek o kargayı kandırmaya çalışır. Optimizasyon açısından, kargalar arama ortamında araştırıcıdır, mevcut ortam arama alanıdır, ortamın her konumu uygun bir çözüme karşılık gelir, besin kaynağının kalitesi objektif (uygunluk) fonksiyonudur ve ortamın en iyi besin kaynağı problemin en iyi çözümüdür (Global çözüm). Bu benzerliklere dayanarak, KAA, optimizasyon problemlerinin çözümünü bulmak için kargaların akıllı davranışlarını simüle etmeye çalışır.

\section{Materyal ve Metot}

\subsection{Karga Arama Algoritması (KAA)}

Kargaların diğer kuşları izlediği, diğer kuşların yiyeceklerini nereye sakladıklarını gözlemlediği ve yiyeceğin sahibi gittikten sonra çaldıkları bilinmektedir. Bir karga hırsızlık yaptıysa, gelecekteki bir kurban olmamak için saklanma yerlerini değiştirmek gibi ekstra önlemler alırlar. Aslında, bir hırsızın davranışını tahmin etmek için kendi hırsız olma deneyimlerini kullanırlar ve önbelleklerini çalınmaktan korumak için en güvenli yolu belirleyebilirler. Bu makalede, daha önce bahsedilen akıllı davranışlara dayalı olarak, popülasyon tabanlı bir metasezgisel algoritma olan KAA anlatılmış ve geliştirilmiştir. KAA ilkeleri şu şekilde sıralanmıştır:

\section{$\checkmark \quad$ Kargalar sürü şeklinde yaşar. \\ $\checkmark \quad$ Kargalar saklandıkları yerin konumunu ezberler. \\ $\checkmark \quad$ Kargalar hırsızlık yapmak için birbirini takip eder. \\ $\checkmark$ Kargalar, önbelleklerini bir olasılıkla çalınmaktan korur}

Bir dizi kargayı içeren $d$ boyutlu bir ortamın olduğu varsayılmaktadır. Kargaların sayısı (sürü boyutu) $N^{\prime}$ dir ve karga $i$ 'nin arama uzayındaki zaman (yineleme) iter'deki konumu bir vektör $x^{i, \text { iter }}\left(i=1,2, \ldots, N\right.$; iter $=1,2, \ldots$, iter $\left._{\text {max }}\right)$ burada $\mathrm{x}^{\mathrm{i}, \text { iter }}=$ $\left[\mathrm{x}^{\mathrm{i}, \text { iter }}{ }_{1}, \mathrm{x}^{\mathrm{i}, \text { iter }}{ }_{2}, \ldots, \mathrm{x}^{\mathrm{i}, \text { iter }}{ }_{\mathrm{d}}\right]$ ve iter $_{\max }$, maksimum yineleme sayısıdır. Her karganın saklandığ yerin hafızaya alındı $\breve{g} 1$ bir hafızası vardır. Yineleme iterinde, karga $i$ 'nin saklanma yeri $m^{i \text {,iter }}$ ile gösterilir. $\mathrm{Bu}$, karganın şimdiye kadar elde ettiği en iyi pozisyondur. Her karganın hafızası, en iyi deneyiminin konumunu ezberlemiştir. Kargalar çevrede hareket eder ve daha iyi besin kaynakları ararlar (saklanma yerleri).

Varsayalımki iter yinelemesinde, karga $j$ 'nin saklanma yerini yani $m^{j, i t e r}$ 'i ziyaret etmek istediğini varsayalım. Bu yinelemede, karga $i$, karga $j$ 'nin saklanma yerine yaklaşmak için karga j'yi takip etmeye karar verir. Bu durumda, iki durum olabilir:

Durum 1: Karga $j$, karga $i$ 'nin kendisini takip ettiğini bilmiyordur. Bir sonuç olarak, karga $i$, karga $j$ 'nin saklandığı yere yaklaşacaktır. Bu durumda, karga $i$ 'nin yeni konumu aşağıdaki gibi elde edilir:

$$
x^{i, i t e r+1}=x^{i, i t e r}+r_{i} \times f l^{i, i t e r} \times\left(m^{j, i t e r}-x^{i, i t e r}\right)
$$

Burada $r_{i}$, 0 ile 1 arasında düzgün dağılıma sahip rastgele bir sayıdır ve $f l^{i, t e r}$, yinelemede karga $i$ 'nin uçuş uzunluğunu gösterir.

Durum 2: Karga $j$, karga $i$ 'nin onu takip ettiğini biliyordur. Sonuç olarak, karga $j$, önbelleğini çalınmaktan korumak için, arama uzayının başka bir konumuna giderek karga i'yi kandıracaktır.

$$
\begin{aligned}
& x^{i, \text { iter }+1}= \\
& \begin{cases}x^{i, i t e r}+r_{i} \times f l^{i, i t e r} \times\left(m^{j, i t e r}-x^{i, i t e r}\right) & r_{j} \geq A P^{i, i t e r} \\
\text { Rastgele bir pozisyon } & \text { aksi halde }\end{cases}
\end{aligned}
$$

Burada $r_{i}$, 0 ile 1 arasında düzgün dağılıma sahip rastgele bir sayıdır ve $A P^{j, i t e r}$, iter yinelemesinde $j$ kargasının farkındalık olasılığını belirtir.

Şekil 1 bu durumun şemasını ve $f l^{\prime}$ in arama kabiliyeti üzerindeki etkisini göstermektedir. Küçük $f l$ değerleri yerel aramaya yol açar ( $x^{i, i t e r}$ yakınlarında) ve büyük değerler genel aramaya yol açar ( $x^{i, i t e r}$ 'den uzak). Şekil 1 (a)'da gösterildiği gibi, $f l$ değeri 1'den küçük seçilirse, karga $i$ 'nin bir sonraki konumu, $\mathrm{x}^{\mathrm{i}, \text { iter }}$ ve $\mathrm{m}^{\mathrm{j}, \text { iter }}$ arasındaki kısa çizgi üzerindedir. Şekil 1(b)'nin gösterdiği gibi, $f l$ değeri 1'den fazla seçilirse, karga i'nin bir sonraki konumu, $\mathrm{m}^{\mathrm{j}}$,iter'i aşabilecek olan çizgi çizgisindedir. 


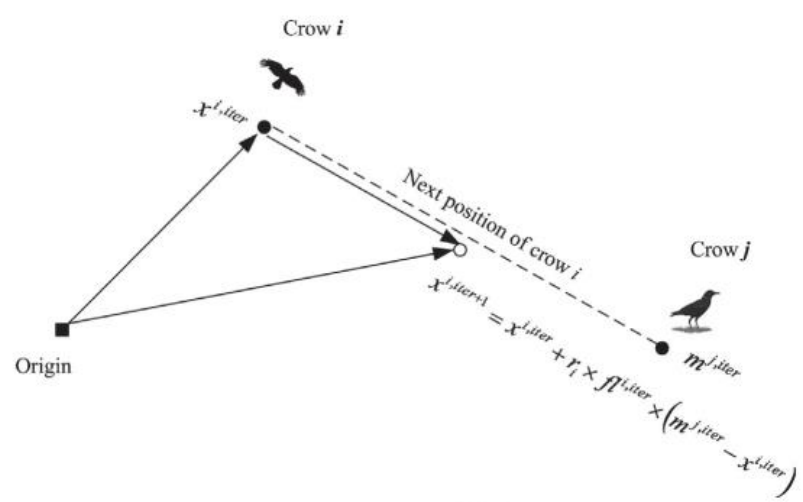

(a) $f l<1$

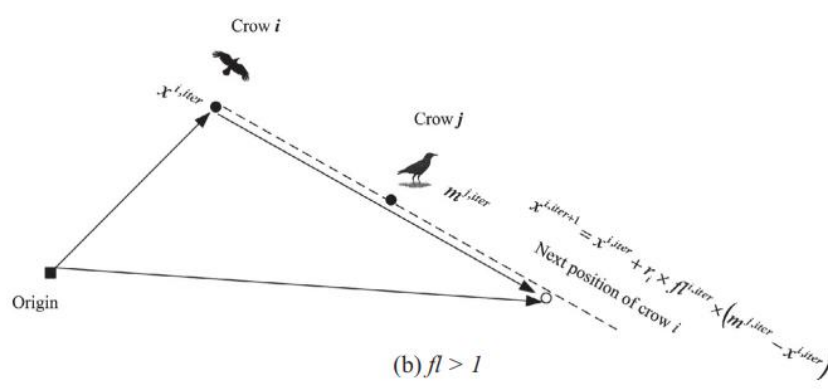

Şekil 1. KAA'da durum 1 'in akış şeması (a) $f l<1$ ve (b) $f l>$ 1. Karga $i$, çizgideki her konuma gidebilir (Askarzadeh, 2016).

Metasezgisel algoritmalar, çeşitlendirme ve yoğunlaştırma arasında iyi bir denge sağlamalıdır. KAA'da, yoğunlaştırma ve çeşitlendirme esas olarak farkındalık olasılığı (AP) parametresi tarafından kontrol edilir. Farkındalık olasılık değerinin düşürülmesiyle, KAA, aramayı bu bölgede mevcut iyi bir çözümün bulunduğu yerel bir bölgede yürütme eğilimindedir. Sonuç olarak, küçük AP değerleri kullanmak yoğunlaşmayı arttırır. Öte yandan, farkındalık olasılık değerinin artmasıyla, mevcut iyi çözümlerin çevresini arama olasılığı azalır ve KAA, arama alanını küresel ölçekte keşfetmeye (randomizasyon) yönelir. Sonuç olarak, AP'nin büyük değerlerinin kullanılması çeşitliliği artırmaktadır.

KAA'nın sözde kodu Şekil 2'de gösterilmiştir

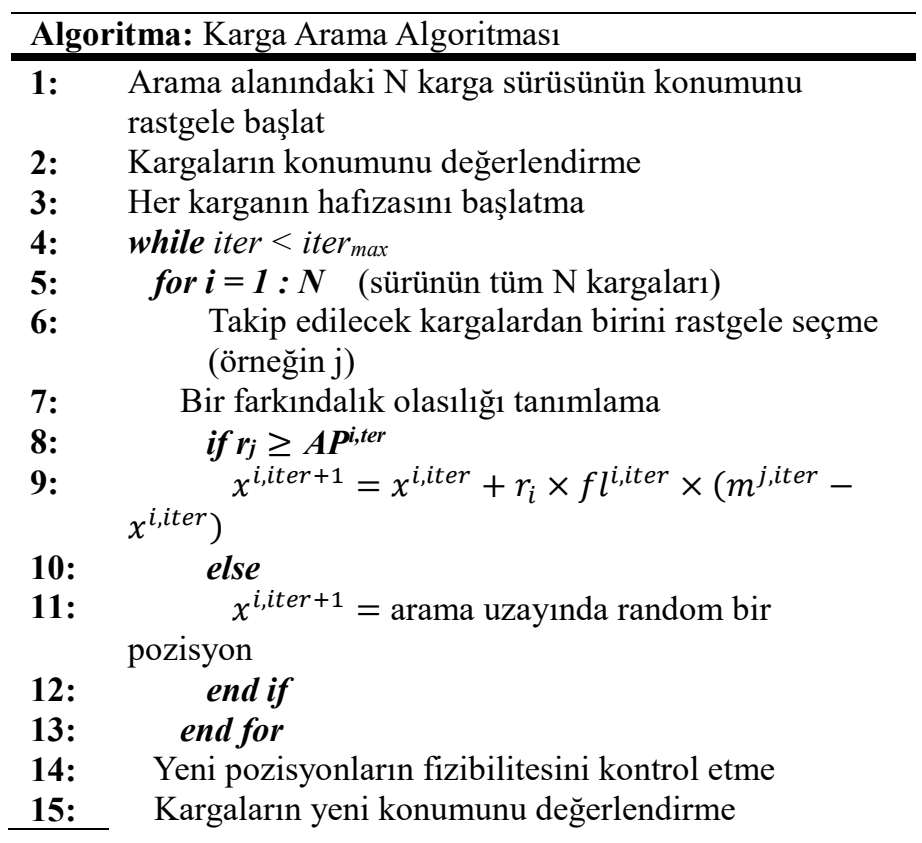

\section{6: Kargaların hafizasını güncelleme \\ 17: end while}

Şekil 2. KAA için sözde kod

\section{Araştırma Sonuçları ve Tartışma}

Algoritma kodlamaları Matlab R2014a üzerinde kodlanmıştır. Uygulamalar işlemcisi intel Core i5 1.19Ghz, ram değeri $12 \mathrm{~Gb}$ olan işletim sistemi windows 10 olan bir makine üzerinde çalıştırılmıştır.

10 farklı sürekli optimizasyon problemini içeren CEC-C06 2019 kıyaslama fomksiyonları üzerinde test işlemleri gerçekleştirilmiştir. Bu test fonksiyonlarının seçilmesinin nedeni literatürde son yıllarda geliştirilmiş olmalarıdır. $\mathrm{Bu}$ test fonksiyonları Price ve ark. tarafindan tek bir amaç optimizasyon problemi için geliştirilmiştir (Price et al., 2018). Test fonksiyonları, yıllık optimizasyon yarışmasında kullanılması amaçlanan "100-Number Challenge" olarak bilinir (Abdullah ve Rashid, 2019). CEC-C06 2019 kıyaslama test fonksiyonlarının tanımları Tablo 2'de gösterilmektedir. Tüm test fonksiyonları minimizasyon fonksiyonlarıdır ve ölçeklenebilirdir. İlk üç CEC fonksiyonunun boyutları sirasıyla 9, 16 ve 18'dir. Diğer CEC işlevlerinin boyutu 10'dur. CEC04 ila CEC10 arasındaki işlevler kaydırılır ve döndürülürken, ilk üç CEC fonksiyonu değildir.

KAA' nın performansı en iyi, en kötü, ortalama ve standart sapma hesaplamaları yapılarak gösterilmiştir. Her bir uygulama 20 bağımsız çalışma şeklinde değerlendirilmiştir. Uygulamalarda kullanılan parametre değerleri Tablo 1 de gösterilmiştir. $f l$ parametre ayarı için 5 farklı değer seçilmiştir. Bu değerlerin sonuç üzerindeki etkisi bu makalede araştırılmıştır. Sonuçlar Tablolar 3 7'de gösterilmiştir. Tablo 8 de farklı fl parametre değerlerine göre ortalama sonuçlarının karşıllaştırması gösterilmiştir. Tablo 9 ve Tablo 10'da ise KAA, DA (Mirjalili, 2015) ve WOA(Mirjalili and Lewis, 2016) sezgisel algoritmaları ortalama ve standart sapma kıyaslama ölçütlerine göre karşılaştırılmıştır.

Tablo 8 sonuçlarına göre, beş farklı fl parametre ortalama değeri karşılaştırılmış ve üstün performans gösteren sonuçlar kalın font ile işaretlenmiştir. Kıyaslama fonksiyonlarının yarısında üstün başarı gösteren fl değeri 4 olarak belirlenmiştir. Diğer değerler daha düşük başarı göstermiştir.

Tablo 9 sonuçlarına göre KAA ile DA ve WOA ortalama sonuçları karşılaştırılmıştır. Üstün performans gösteren sonuçlar kalın font ile işaretlenmiştir. On kıyaslama fonksiyonunun dört tanesinde DA, üç tanesinde KAA ve üç tanesinde de WOA üstün başarı sağlamıştır.

Tablo 1. Parametre ayarlarl

\begin{tabular}{cc}
\hline Parametre & Değer \\
\hline Populasyon sayısı & 50 \\
Boyut & $\{9,10,16,18\}$ \\
Maksimum iterasyon & 500 \\
$A P$ & 0.1 \\
$f l$ & $\{2,4,6,8,10\}$ \\
\hline
\end{tabular}

Tablo 2. CEC-C062019 Test Fonksiyonlarının Tanımlaması 


\begin{tabular}{|c|c|c|c|c|}
\hline ID & Functions & Dimension & Range & $f_{\text {min }}$ \\
\hline 1 & $\begin{array}{c}\text { Storn's Chebyshev } \\
\text { Polynomial Fitting } \\
\text { Problem }\end{array}$ & 9 & $\begin{array}{c}{[-8192,} \\
8192]\end{array}$ & 1 \\
\hline 2 & $\begin{array}{l}\text { Inverse Hilbert } \\
\text { Matrix Problem }\end{array}$ & 16 & $\begin{array}{c}{[-16384,} \\
16384]\end{array}$ & 1 \\
\hline 3 & $\begin{array}{c}\text { Lennard-Jones } \\
\text { Minimum Energy } \\
\text { Cluster }\end{array}$ & 18 & {$[-4,4]$} & 1 \\
\hline 4 & Rastrigin's Function & 10 & {$[-100,100]$} & 1 \\
\hline 5 & Griewank's Function & 10 & {$[-100,100]$} & 1 \\
\hline 6 & Weierstrass Function & 10 & {$[-100,100]$} & 1 \\
\hline 7 & $\begin{array}{l}\text { Modified Schwefel's } \\
\text { Function }\end{array}$ & 10 & {$[-100,100]$} & 1 \\
\hline 8 & $\begin{array}{c}\text { Expanded Schaffer's } \\
\text { F6 Function }\end{array}$ & 10 & {$[-100,100]$} & 1 \\
\hline 9 & Happy Cat Function & 10 & {$[-100,100]$} & 1 \\
\hline 10 & Ackley Function & 10 & {$[-100,100]$} & 1 \\
\hline
\end{tabular}

Tablo 3. KAA 'nin CEC-C06 2019 test fonksiyonlart üzerindeki performans sonuçları $(f l=2)$

\begin{tabular}{|c|c|c|c|c|}
\hline $\begin{array}{c}\text { Fonksiyon } \\
\text { No }\end{array}$ & En iyi & En kötü & Ortalama & $\begin{array}{c}\text { Standart } \\
\text { Sapma }\end{array}$ \\
\hline CEC01 & $1,99 \mathrm{E}+06$ & $4,97 \mathrm{E}+08$ & $8,57 \mathrm{E}+07$ & $1,15 \mathrm{E}+08$ \\
\hline$C E C 02$ & $7,75 \mathrm{E}+03$ & $2,41 \mathrm{E}+04$ & $1,66 \mathrm{E}+04$ & $4,69 \mathrm{E}+03$ \\
\hline$C E C 03$ & $1,27 \mathrm{E}+01$ & $1,27 \mathrm{E}+01$ & $1,27 \mathrm{E}+01$ & $9,98 \mathrm{E}-04$ \\
\hline$C E C 04$ & $1,23 \mathrm{E}+04$ & $5,21 \mathrm{E}+04$ & $2,47 \mathrm{E}+04$ & $8,20 \mathrm{E}+03$ \\
\hline$C E C 05$ & $3,97 \mathrm{E}+00$ & $1,04 \mathrm{E}+01$ & $7,03 \mathrm{E}+00$ & $1,62 \mathrm{E}+00$ \\
\hline$C E C 06$ & $1,33 \mathrm{E}+01$ & $1,68 \mathrm{E}+01$ & $1,51 \mathrm{E}+01$ & $9,75 \mathrm{E}-01$ \\
\hline$C E C 07$ & $1,38 \mathrm{E}+03$ & $2,72 \mathrm{E}+03$ & $1,99 \mathrm{E}+03$ & $3,06 \mathrm{E}+02$ \\
\hline$C E C 08$ & $6,68 \mathrm{E}+00$ & $9,11 \mathrm{E}+00$ & $8,01 \mathrm{E}+00$ & $5,61 \mathrm{E}-01$ \\
\hline$C E C 09$ & $2,81 \mathrm{E}+03$ & $8,87 \mathrm{E}+03$ & $5,47 \mathrm{E}+03$ & $1,45 \mathrm{E}+03$ \\
\hline$C E C 10$ & $2,06 \mathrm{E}+01$ & $2,14 \mathrm{E}+01$ & $2,11 \mathrm{E}+01$ & $1,66 \mathrm{E}-01$ \\
\hline
\end{tabular}

\begin{tabular}{|l|l|l|l|l|}
\hline$C E C 04$ & $8,94 \mathrm{E}+03$ & $3,70 \mathrm{E}+04$ & $2,40 \mathrm{E}+04$ & $7,57 \mathrm{E}+03$ \\
\hline$C E C 05$ & $4,46 \mathrm{E}+00$ & $1,20 \mathrm{E}+01$ & $7,14 \mathrm{E}+00$ & $1,77 \mathrm{E}+00$ \\
\hline$C E C 06$ & $1,38 \mathrm{E}+01$ & $1,76 \mathrm{E}+01$ & $1,58 \mathrm{E}+01$ & $1,06 \mathrm{E}+00$ \\
\hline$C E C 07$ & $1,61 \mathrm{E}+03$ & $2,54 \mathrm{E}+03$ & $2,01 \mathrm{E}+03$ & $2,76 \mathrm{E}+02$ \\
\hline$C E C 08$ & $7,54 \mathrm{E}+00$ & $8,98 \mathrm{E}+00$ & $8,38 \mathrm{E}+00$ & $3,76 \mathrm{E}-01$ \\
\hline$C E C 09$ & $2,50 \mathrm{E}+03$ & $8,66 \mathrm{E}+03$ & $5,70 \mathrm{E}+03$ & $1,54 \mathrm{E}+03$ \\
\hline$C E C 10$ & $2,08 \mathrm{E}+01$ & $2,13 \mathrm{E}+01$ & $2,11 \mathrm{E}+01$ & $1,47 \mathrm{E}-01$ \\
\hline
\end{tabular}

Tablo 6. KAA 'nin CEC-C06 2019 test fonksiyonlarl üzerindeki performans sonuçları $(f l=8)$

\begin{tabular}{|c|c|c|c|c|}
\hline $\begin{array}{c}\text { Fonksiyon } \\
\text { No }\end{array}$ & En iyi & En kötü & Ortalama & $\begin{array}{c}\text { Standart } \\
\text { Sapma }\end{array}$ \\
\hline$C E C 01$ & $4,68 \mathrm{E}+08$ & $6,27 \mathrm{E}+09$ & $1,90 \mathrm{E}+09$ & $1,32 \mathrm{E}+09$ \\
\hline$C E C 02$ & $5,98 \mathrm{E}+03$ & $2,11 \mathrm{E}+04$ & $1,35 \mathrm{E}+04$ & $4,41 \mathrm{E}+03$ \\
\hline$C E C 03$ & $1,27 \mathrm{E}+01$ & $1,27 \mathrm{E}+01$ & $1,27 \mathrm{E}+01$ & $1,03 \mathrm{E}-03$ \\
\hline$C E C 04$ & $9,10 \mathrm{E}+03$ & $3,90 \mathrm{E}+04$ & $2,56 \mathrm{E}+04$ & $8,00 \mathrm{E}+03$ \\
\hline$C E C 05$ & $4,77 \mathrm{E}+00$ & $1,06 \mathrm{E}+01$ & $7,58 \mathrm{E}+00$ & $1,38 \mathrm{E}+00$ \\
\hline$C E C 06$ & $1,11 \mathrm{E}+01$ & $1,73 \mathrm{E}+01$ & $1,52 \mathrm{E}+01$ & $1,43 \mathrm{E}+00$ \\
\hline$C E C 07$ & $1,43 \mathrm{E}+03$ & $2,97 \mathrm{E}+03$ & $2,09 \mathrm{E}+03$ & $3,61 \mathrm{E}+02$ \\
\hline$C E C 08$ & $7,83 \mathrm{E}+00$ & $9,39 \mathrm{E}+00$ & $8,48 \mathrm{E}+00$ & $4,80 \mathrm{E}-01$ \\
\hline$C E C 09$ & $3,06 \mathrm{E}+03$ & $7,89 \mathrm{E}+03$ & $5,61 \mathrm{E}+03$ & $1,07 \mathrm{E}+03$ \\
\hline$C E C 10$ & $2,07 \mathrm{E}+01$ & $2,14 \mathrm{E}+01$ & $2,11 \mathrm{E}+01$ & $1,55 \mathrm{E}-01$ \\
\hline
\end{tabular}

Tablo 7. KAA 'nin CEC-C06 2019 test fonksiyonlart üzerindeki performans sonuçları $(f l=10)$

\begin{tabular}{|c|c|c|c|c|}
\hline $\begin{array}{c}\text { Fonksiyon } \\
\text { No }\end{array}$ & En iyi & En kötü & Ortalama & $\begin{array}{c}\text { Standart } \\
\text { Sapma }\end{array}$ \\
\hline$C E C 01$ & $5,10 \mathrm{E}+08$ & $1,09 \mathrm{E}+10$ & $3,24 \mathrm{E}+09$ & $2,71 \mathrm{E}+09$ \\
\hline$C E C 02$ & $8,03 \mathrm{E}+03$ & $2,50 \mathrm{E}+04$ & $1,40 \mathrm{E}+04$ & $3,66 \mathrm{E}+03$ \\
\hline$C E C 03$ & $1,27 \mathrm{E}+01$ & $1,27 \mathrm{E}+01$ & $1,27 \mathrm{E}+01$ & $1,09 \mathrm{E}-03$ \\
\hline$C E C 04$ & $1,05 \mathrm{E}+04$ & $4,22 \mathrm{E}+04$ & $2,56 \mathrm{E}+04$ & $8,75 \mathrm{E}+03$ \\
\hline$C E C 05$ & $3,95 \mathrm{E}+00$ & $9,96 \mathrm{E}+00$ & $7,23 \mathrm{E}+00$ & $1,59 \mathrm{E}+00$ \\
\hline$C E C 06$ & $1,17 \mathrm{E}+01$ & $1,73 \mathrm{E}+01$ & $1,56 \mathrm{E}+01$ & $1,33 \mathrm{E}+00$ \\
\hline$C E C 07$ & $1,51 \mathrm{E}+03$ & $2,52 \mathrm{E}+03$ & $2,15 \mathrm{E}+03$ & $2,77 \mathrm{E}+02$ \\
\hline$C E C 08$ & $7,20 \mathrm{E}+00$ & $8,63 \mathrm{E}+00$ & $8,01 \mathrm{E}+00$ & $4,15 \mathrm{E}-01$ \\
\hline$C E C 09$ & $3,13 \mathrm{E}+03$ & $8,10 \mathrm{E}+03$ & $5,45 \mathrm{E}+03$ & $1,39 \mathrm{E}+03$ \\
\hline$C E C 10$ & $2,07 \mathrm{E}+01$ & $2,14 \mathrm{E}+01$ & $2,11 \mathrm{E}+01$ & $1,49 \mathrm{E}-01$ \\
\hline
\end{tabular}

Tablo 8. KAA 'nin farkl fl değerlerine göre ortalama sonuçlarının karşılaş̧tırması

\begin{tabular}{|c|c|c|c|c|}
\hline $\begin{array}{c}\text { Fonksiyon } \\
\text { No }\end{array}$ & En iyi & En kötü & Ortalama & $\begin{array}{c}\text { Standart } \\
\text { Sapma }\end{array}$ \\
\hline CEC01 & $4,61 \mathrm{E}+06$ & $5,04 \mathrm{E}+08$ & $1,37 \mathrm{E}+08$ & $1,58 \mathrm{E}+08$ \\
\hline CEC02 & $1,00 \mathrm{E}+04$ & $2,19 \mathrm{E}+04$ & $1,54 \mathrm{E}+04$ & $3,31 \mathrm{E}+03$ \\
\hline CEC03 & $1,27 \mathrm{E}+01$ & $1,27 \mathrm{E}+01$ & $1,27 \mathrm{E}+01$ & $8,24 \mathrm{E}-04$ \\
\hline CEC04 & $7,47 \mathrm{E}+03$ & $4,20 \mathrm{E}+04$ & $2,58 \mathrm{E}+04$ & $8,13 \mathrm{E}+03$ \\
\hline$C E C 05$ & $4,09 \mathrm{E}+00$ & $9,63 \mathrm{E}+00$ & $7,01 \mathrm{E}+00$ & $1,35 \mathrm{E}+00$ \\
\hline$C E C 06$ & $1,43 \mathrm{E}+01$ & $1,73 \mathrm{E}+01$ & $1,55 \mathrm{E}+01$ & $7,99 \mathrm{E}-01$ \\
\hline$C E C 07$ & $1,05 \mathrm{E}+03$ & $2,50 \mathrm{E}+03$ & $1,92 \mathrm{E}+03$ & $4,20 \mathrm{E}+02$ \\
\hline$C E C 08$ & $6,80 \mathrm{E}+00$ & $8,88 \mathrm{E}+00$ & $8,07 \mathrm{E}+00$ & $5,13 \mathrm{E}-01$ \\
\hline$C E C 09$ & $3,43 \mathrm{E}+03$ & $9,90 \mathrm{E}+03$ & $5,71 \mathrm{E}+03$ & $1,49 \mathrm{E}+03$ \\
\hline$C E C 10$ & $2,06 \mathrm{E}+01$ & $2,13 \mathrm{E}+01$ & $2,10 \mathrm{E}+01$ & $1,58 \mathrm{E}-01$ \\
\hline
\end{tabular}

Tablo 5. KAA 'nın CEC-C06 2019 test fonksiyonlart üzerindeki performans sonuçları $(f l=6)$

\begin{tabular}{|c|c|c|c|c|}
\hline $\begin{array}{c}\text { Fonksiyon } \\
\text { No }\end{array}$ & En iyi & En kötü & Ortalama & $\begin{array}{c}\text { Standart } \\
\text { Sapma }\end{array}$ \\
\hline$C E C 01$ & $1,15 \mathrm{E}+08$ & $2,98 \mathrm{E}+09$ & $8,18 \mathrm{E}+08$ & $7,09 \mathrm{E}+08$ \\
\hline$C E C 02$ & $6,86 \mathrm{E}+03$ & $2,19 \mathrm{E}+04$ & $1,34 \mathrm{E}+04$ & $3,96 \mathrm{E}+03$ \\
\hline$C E C 03$ & $1,27 \mathrm{E}+01$ & $1,27 \mathrm{E}+01$ & $1,27 \mathrm{E}+01$ & $6,65 \mathrm{E}-04$ \\
\hline
\end{tabular}

\begin{tabular}{|c|c|c|c|c|c|}
\hline $\begin{array}{c}\text { Fonksiyon } \\
\text { No }\end{array}$ & $f l=2$ & $f l=4$ & $f l=6$ & $f l=8$ & $f l=10$ \\
\hline CECO1 & $8,57 \mathrm{E}+07$ & $1,37 \mathrm{E}+08$ & $8,18 \mathrm{E}+08$ & $1,90 \mathrm{E}+09$ & $3,24 \mathrm{E}+09$ \\
\hline CEC02 & $6 \mathrm{E}+04$ & $54 \mathrm{E}+04$ & $1,34 \mathrm{E}+04$ & $1,35 \mathrm{E}+04$ & $40 \mathrm{E}+04$ \\
\hline $\mathrm{CECO} 3$ & $1,27 \mathrm{E}+01$ &, $27 \mathrm{E}+01$ & $1,27 \mathrm{E}+01$ & $1,27 \mathrm{E}+01$ & $1,27 \mathrm{E}+01$ \\
\hline & $2,47 \mathrm{E}+04$ & $2,58 \mathrm{E}+04$ & $2,40 E+04$ & $2,56 \mathrm{E}+04$ & $2,56 \mathrm{E}+04$ \\
\hline & $7,03 \mathrm{E}+00$ & $7,01 E+00$ & 7,14E+00 & $7,58 \mathrm{E}+00$ & $7,23 \mathrm{E}+00$ \\
\hline C06 & $1,51 \mathrm{E}+01$ & $1,55 \mathrm{E}+01$ & $1,58 \mathrm{E}+01$ & $1,52 \mathrm{E}+01$ & $1,56 \mathrm{E}+01$ \\
\hline$E C 07$ & $1,99 \mathrm{E}+03$ & $1,92 \mathrm{E}+03$ & $2,01 \mathrm{E}+03$ & $2,09 \mathrm{E}+03$ & $2,15 \mathrm{E}+03$ \\
\hline$E C 08$ & $8,01 E$ & 00 & $8,38 \mathrm{~F}$ & $8,48 \mathrm{E}+00$ & $8,01 \mathrm{E}+00$ \\
\hline & $5,47 \mathrm{E}+03$ & $5,71 \mathrm{~F}$ & $5,70 \mathrm{E}$ & $5,61 \mathrm{E}+03$ & $5,45 \mathrm{E}+03$ \\
\hline EC10 & $2,11 \mathrm{E}+01$ & $2,10 \mathrm{E}+01$ & $2,11 \mathrm{E}+01$ & $2,11 \mathrm{E}+01$ & $2,11 \mathrm{E}+01$ \\
\hline
\end{tabular}


Tablo 9. IKAA'nın ve diğer metasezgisel algoritmaların ortalama karşılaştırma sonuçları

\begin{tabular}{|c|c|c|c|}
\hline $\begin{array}{c}\text { Fonksiyon } \\
\text { No }\end{array}$ & $D A$ & WOA & KAA \\
\hline CEC01 & $543 \times 10^{8}$ & $411 \times 10^{8}$ & $\mathbf{1 , 3 7 E + 0 8}$ \\
\hline CEC02 & 78.0368 & $\mathbf{1 7 . 3 4 9 5}$ & $1,54 \mathrm{E}+04$ \\
\hline CEC03 & 13.7026 & 13.7024 & $\mathbf{1 , 2 7 E + 0 1}$ \\
\hline CEC04 & $\mathbf{3 4 4 . 3 5 6 1}$ & 394.6754 & $2,58 \mathrm{E}+04$ \\
\hline CEC05 & $\mathbf{2 . 5 5 7 2}$ & 2.7342 & $7,01 \mathrm{E}+00$ \\
\hline CEC06 & $\mathbf{9 . 8 9 5 5}$ & 10.7085 & $1,55 \mathrm{E}+01$ \\
\hline CEC07 & 578.9531 & $\mathbf{4 9 0 . 6 8 4 3}$ & $1,92 \mathrm{E}+03$ \\
\hline CEC08 & $\mathbf{6 . 8 7 3 4}$ & 6.909 & $8,07 \mathrm{E}+00$ \\
\hline CEC09 & 6.0467 & $\mathbf{5 . 9 3 7 1}$ & $5,71 \mathrm{E}+03$ \\
\hline CEC10 & 21.2604 & 21.2761 & $\mathbf{2 , 1 0 E}+\mathbf{0 1}$ \\
\hline
\end{tabular}

Tablo 10. IKAA'nın ve diğer metasezgisel algoritmaların standart sapma karşılaştırma sonuçları

\begin{tabular}{|c|c|c|c|}
\hline $\begin{array}{c}\text { Fonksiyon } \\
N o\end{array}$ & $D A$ & WOA & KAA \\
\hline CEC01 & $669 \times 10^{8}$ & $542 \times 10^{8}$ & $\mathbf{1 , 5 8 E + 0 8}$ \\
\hline CEC02 & 87.7888 & $\mathbf{0 . 0 0 4 5}$ & $3,31 \mathrm{E}+03$ \\
\hline CEC03 & 0.0007 & $\mathbf{0 . 0}$ & $8,24 \mathrm{E}-04$ \\
\hline CEC04 & 414.0982 & $\mathbf{2 4 8 . 5 6 2 7}$ & $8,13 \mathrm{E}+03$ \\
\hline CEC05 & 0.3245 & $\mathbf{0 . 2 9 1 7}$ & $1,35 \mathrm{E}+00$ \\
\hline CEC06 & 1.6404 & 1.0325 & $\mathbf{7 , 9 9 E - 0 1}$ \\
\hline CEC07 & 329.3983 & $\mathbf{1 9 4 . 8 3 1 8}$ & $4,20 \mathrm{E}+02$ \\
\hline CEC08 & 0.5015 & $\mathbf{0 . 4 2 6 9}$ & $5,13 \mathrm{E}-01$ \\
\hline$C E C 09$ & 2.871 & $\mathbf{1 . 6 5 6 6}$ & $1,49 \mathrm{E}+03$ \\
\hline$C E C 10$ & 0.1715 & $\mathbf{0 . 1 1 1 1}$ & $1,58 \mathrm{E}-01$ \\
\hline
\end{tabular}

\section{Sonuç}

Mevcut eğilim, zor problemlerin üstesinden gelmek için doğadan ilham alan metasezgisel algoritmaları kullanmaktır. Karga Arama Algoritması (KAA) da bu tarz algoritmalardan bir tanesidir. Bir karga sürüsü, optimizasyon süreciyle pek çok benzerliği olan bir davranış sergilerler. $\mathrm{Bu}$ davranışa göre kargalar fazla yiyeceklerini ortamın belirli konumlarında (saklanma yerlerinde) saklarlar ve depolanan yiyecekleri gerektiğinde geri bulurlar. $\mathrm{Bu}$ davranıştan esinlenilerek KAA oluşturulmuştur. KAA sürekli optimizasyon problemlerini çözsede detaylı bir parametre analizi yapılmamıştır. $\mathrm{Bu}$ çalışmada $f l$ parametre değerinin arama kabiliyeti üzerindeki etkisi araştırılmıştır. Çünkü küçük $f l$ değerleri yerel aramaya yol açarken büyük değerler genel aramaya yol açar. Beş farklı fl değerinin on farklı CEC 2019 kıyaslama fonksiyonu üzerinde testleri gerçekleştirilmiştir. En başarılı fl değeri dört olarak tespit edilmiştir. Bu da göstermiştir ki yerel arama kabiliyeti performans üzerindeki etkisi daha fazladır. En başarılı KAA sonuçları literatürden seçilen DA ve WOA algoritmaları ile karşılaştırlmıştır. Sonuç olarak KAA etkili algoritmalardan biri olduğu ispatlanmıştır.

Geleceğe dair çalışmalarda KAA nın arama yeteneğinin farklı teknikler kullanılarak geliştirilmesi düşünülmektedir. CEC 2017 test fonksiyonlarında başarısı gösterilmesi düşünülmektedir.

\section{Kaynakça}

Abdullah, J. M., Rashid, A.T. "Fitness Dependent Optimizer: Inspired by the Bee Swarming Reproductive Process," in IEEE Access, vol. 7, pp. 43473-43486, 2019, doi: 10.1109/ACCESS.2019.2907012.

Askarzadeh, A., 2016. A novel metaheuristic method for solving constrained engineering optimization problems: Crow search algorithm, Computers and Structures, 169, 1-12.

Mirjalili, S., "Dragonfly algorithm: A new meta-heuristic optimization technique for solving single-objective, discrete, and multi-objective problems," Neural Comput. Appl., vol. 27, no. 4, pp. 1053-1073, May 2015.

Mirjalili, S., Lewis, A., "The whale optimization algorithm," Adv. Eng. Softw., vol. 95, pp. 51-67, May 2016.

Price, K. V., Awad, N. H., Ali, M. Z., Suganthan, P. N., “The 100digit challenge: Problem definitions and evaluation criteria for the 100-digit challenge special session and competition on single objective numerical optimization," School Elect. Electron. Eng., Nanyang Technol. Univ., Singapore, Tech. Rep., Nov. 2018.

Yang, X.S, 2011. Metaheuristic optimization, Scholarpedia 2011; 611472. 